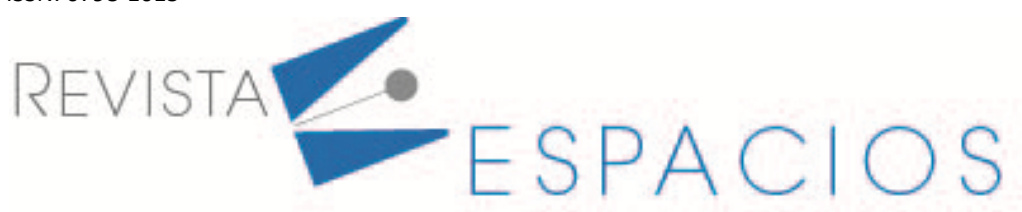

\title{
Mensuração do comportamento empreendedor como norteador de competitividade em startups na fase inicial do negócio
}

\section{Measurement of entrepreneurial behavior as a competitiveness driver in startups in the initial phase of the business}

\author{
MICHELIN, Cláudia de Freitas ${ }^{1}$ \\ MINELLO, Italo Fernando ${ }^{2}$ \\ SILUK, Julio Cezar Mairesse ${ }^{3}$ \\ SANTOS, Jordana Rech Graciano ${ }^{4}$ \\ GERHARDT, Vinícius Jaques ${ }^{5}$ \\ STIELER, Eduarda Grasel ${ }^{6}$
}

\begin{abstract}
Resumo
Esse estudo exploratório e descritivo objetivou mensurar o comportamento empreendedor de gestores na fase inicial do ciclo organizacional em startups incubadas, enfatizando que o resultado pode ser norteador de competitividade. Com base em uma pesquisa teórica-empírica fundamentada no indivíduo empreendedor, os dados de abordagem quantitativa foram tratados utilizando elementos da Análise Hierárquica de Processos. Como resultados destaca-se que os gestores pesquisados apresentam indices elevados de características do comportamento empreendedor (CCE) significando que os negócios geridos por eles foram considerados potencialmente ou plenamente competitivos na perspectiva comportamental.

Palavras-chave: competitividade, comportamento empreendedor, sistema de mensuração de desempenho
\end{abstract}

\begin{abstract}
This study is characterized as exploratory and descriptive, aimed to measure the entrepreneurial behavior of managers in the initial phase of the organizational cycle in incubated startups, emphasizing that the result can be a guide for competitiveness. Based on theoretical-empirical research the data were treated using elements of the Hierarchical Analysis of Processes. As a result, the surveyed managers have high indices of characteristics of entrepreneurial behavior (CCE) highlighting that the businesses they manage were considered potentially or fully competitive in the behavioral perspective. Keywords: competitiveness, entrepreneurial behavior, performance measurement system
\end{abstract}

\footnotetext{
${ }^{1}$ Docente, Departamento de Ciências Contábeis, Universidade Federal de Santa Maria, claudia.michelin@ufsm.br

2 Docente, Departamento de Administração, Universidade Federal de Santa Maria, minelloif@gmail.com

${ }^{3}$ Docente, Departamento de Engenharia de Produção e Sistemas, Universidade Federal de Santa Maria, jsiluk@ufsm.br

${ }^{4}$ Pós-Graduanda em Engenharia de Produção, Departamento de Engenharia de Produção e Sistemas, Universidade Federal de Santa Maria, jordana_rech@hotmail.com

${ }^{5}$ Pós-Graduando em Engenharia de Produção, Departamento de Engenharia de Produção e Sistemas, Universidade Federal de Santa Maria, viniciusgerhardt92@gmail.com

${ }^{6}$ Graduanda em Ciências Contábeis, Departamento de Ciências Contábeis, Universidade Federal de Santa Maria, dudagrasel@gmail.com
} 


\section{Introdução}

$\mathrm{Na}$ conjuntura ampla das organizações, destaca-se as startups, que são empresas que têm alterado drasticamente as indústrias tradicionais, principalmente aquelas que trabalham com produtos e serviços inteligentes (Hahn, 2020; Neamţu, Bejinaru, \& Hapenciuc, 2020). Esse tipo de organização é considerado intensiva em conhecimentos pois se caracteriza como modelos de negócios repetitivos, escaláveis e inovadores. As startups são reconhecidas por contribuírem economicamente de forma significativa em diversas regiões ou países, gerando empregos e outros benefícios necessários às comunidades nas quais estão inseridas (Colombo \& Grilli, 2005), sendo que grande parte delas desfrutam de estruturas de incubadoras tecnológicas em seus períodos iniciais de criação do negócio.

No Brasil, o processo de incubação de empresas de base tecnológica tem auxiliado no processo de desenvolvimento desses negócios. O maior banco de dados de startups do país, o StartupBase, possui registradas 6119 empresas, sendo 4,69\% delas situadas no estado do Rio Grande do Sul, local onde a pesquisa foi realizada com grande parte delas integrando as 369 incubadoras de empresas em funcionamento no Brasil (Associação Nacional de Entidades Promotoras de Empreendimentos Inovadores [ANPROTEC] 2016).

Os empreendedores à frente dessas empresas têm como foco a capitalização e a rentabilidade dos seus negócios a fim de alavancar sua competitividade ao longo do ciclo organizacional do negócio (Antonenko, Lee, \& Kleinheksel, 2014). Também enfrentam riscos consideráveis em relação a credibilidade do modelo de negócios e a viabilidade tecnológica do produto ou serviço (Dutta \& Folta, 2016 ; Nascimento, et al., 2019). Embora em um primeiro momento possa ser necessária a contratação de profissionais para fins específicos no período inicial da empresa, é mais eficiente e seguro que os próprios fundadores possuam habilidades na resolução de problemas unidos aos conhecimentos técnicos necessários ao desenvolvimento do seu negócio, além também de adotar práticas de governança (Kosuri, 2017).

As startups que apresentam estruturas de governança sabem identificar ameaças e oportunidades em ambiente de negócios (Dutta \& Folta, 2016). Contribuindo, o capital intelectual e as características comportamentais dos empreendedores possuem influência crucial nos momentos iniciais do negócio, sendo diretamente responsáveis por seu eventual sucesso ou fracasso (Colombo \& Piva, 2012). Para Siluk, Garlet, Marcuzzo, Michelin, \& Minello (2018), enquanto o capital humano impacta a atividade empreendedora, a habilidade para adquiri-lo reflete as turbulências do ambiente em que se encontra determinado ecossistema econômico-social corporativo. Impulsionados pelas frequentes inovações tecnológicas e desempenho da economia, ecossistemas corporativos tem sido marcados pela rapidez com que se processam as mudanças e essas transformações têm desafiado as organizações a repensar o papel crucial de suas políticas e práticas organizacionais em relação à competitividade (Silveira, Michelin, \& Siluk, 2017; Mitsakis, 2017).

A competitividade de negócios pressupõe uma correspondência entre a estratégia da empresa e suas competências internas com oportunidades externas, e a aceitação e o ajuste da estratégia pelo ambiente em que a empresa concorre, isso proporciona uma vantagem competitiva sustentável diante dos concorrentes, assegurando e aumentando a participação de mercado e gerando lucros (Caseiro \& Coelho, 2018; Maune, 2014). Os mesmos autores ainda dizem que apesar de a competitividade poder ser definida como uma condição enfocando os fatores que levam à ela, também pode ser analisada como uma atitude dos empreendedores que desenvolvem tais negócios.

Essas atitudes são reflexos das características comportamentais definidas como as ações que são necessárias para iniciar e desenvolver uma nova organização (Bird, Schjoedt, \& Baum, 2012), adotando assim uma perspectiva de empreendedorismo que está embutida na racionalidade econômica e focada na criação de 
empreendimentos lucrativos (Gruber \& MacMillan, 2017). No âmbito das startups incubadas, analisar tais características apresenta-se como importante para elevar a competitividade nos negócios.

Os desafios enfrentados pelas empresas ao longo de sua existência requerem educação, experiência e motivação dos gestores no engajamento das estratégias que resultarão em um aumento de desempenho (Peña, 2002). Tendo em vista que a pesquisa focada na atividade empreendedora vem sendo valorizada como uma das principais bases do crescimento econômico e da geração de emprego e renda da atualidade ("GEM: Global Entrepreneurship Monitor," n.d.), verifica-se a necessidade de estudos que tratam do aspecto comportamental por entender que eles afetam significativamente nos resultados dos negócios. Pessoas empreendedoras influenciam a criação de novos produtos com ideias inovadoras baseadas em suas experiências e no ambiente de trabalho (Tripathi, Oivo, Liukkunen, \& Markkula, 2019), portanto, o empreendedorismo é um fenômeno multidimensional que gera muitas contribuições (Figueiredo \& Paiva, 2019).

Diante da contextualização e por considerar pertinente estudar a competitividade em ambientes corporativos, o objetivo desse estudo foi mensurar o comportamento empreendedor de gestores na fase inicial do ciclo organizacional em startups incubadas, enfatizando que o resultado da mensuração pode ser norteador de competitividade.

No sentido de valorar essa forma de pesquisa, esse estudo destaca publicações sobre mensuração da competividade utilizando sistema de mensuração de desempenho (SMD). Os autores Costa, Siluk, Neuenfeldt Júnior, Soliman, \& Nara (2015) pesquisaram a competitividade na esfera industrial e enfatizaram a importância de SMD como uma ferramenta de trabalho, uma vez que permite uma visão detalhada de processos assim como soluções mais rápidas e eficientes sobre qualquer alteração desejada de modo a fomentar novos estudos teóricos e práticos a respeito do assunto pesquisado. Já Scarano, Siluk, Nara, Neuenfeldt Júnior, \& Da Fontoura (2014) pesquisaram a competitividade do setor metal mecânico, levantando primeiramente os fatores críticos de sucesso (FCS) por meio de um diagnóstico, identificando e avaliando através de um SMD os critérios mais relevantes para o setor, deixando nesse sentido sua contribuição. Outra mensuração da competitividade avaliada por um SMD foi explorada na pesquisa desenvolvida por Nora et al. (2016) onde o foco foi a relação da competitividade e inovação no setor de serviços de telecomunicações.

O tema mensuração de desempenho organizacional vem recebendo atenção crescente devido à pressão sofrida pelas empresas por apresentar resultados; neste sentido, o desenvolvimento de um SMD é visto como um fator chave no apoio a gestão, pois pode favorecer a tomada de decisões corretas e a tempo das operações da organização (Siluk, 2007), além de reforçar vantagens em relação aos fatores internos como externos, com foco em melhorias de processo (Marinho \& Cagnin, 2014). O presente estudo também optou pelo método SMD e foi estruturado em cinco partes: (1) aspectos introdutórios, (2) fundamentação teórica, (3) procedimentos metodológicos, (4) discussão dos resultados e (5) conclusões.

\section{Referencial Teórico}

O trabalho fundamentou-se acerca dos temas de competitividade, comportamento empreendedor, ciclo organizacional do negócio com ênfase na fase de criação e sistemas de mensuração de desempenho.

\subsection{Competitividade}

A avaliação da competitividade inclui fatores como inovação, aprendizagem e capacidades empresariais, bem como indicadores de gestão (Poveda-Bautista, García-Melón, \& Baptista, 2013). No nível das empresas, o conhecimento tecnológico pode fundamentar uma empresa competitiva e a busca permanente de inovações pode recriar as condições para que esta se matenha ao longo do tempo. Assim, competitividade não é um 
conceito estático e o dinamismo requerido para a manutenção de posições competitivas requer o investimento em aquisição ou desenvolvimento tecnológico (Bartz, Siluk, \& Bartz, 2014).

A competitividade de uma empresa está relacionada à sua presença contínua no mercado, assim como a sua lucratividade e produtividade (Díaz-Chao, Sainz-González, \& Torrent-Sellens, 2016). Contudo, com a finalidade de ganhar vantagem competitiva, é importante desdobrar as atividades de uma empresa em diversas partes que contribuem de alguma forma para o desempenho final do produto ou serviço. A vantagem competitiva surge quando a empresa desempenha as atividades em cada uma dessas partes de maneira mais eficiente que seus concorrentes, ou de forma que crie maior valor percebido para o cliente (Porter \& Kramer, 2011). Segundo Minello (2010) é possível avaliar a competitividade por meio das ações das pessoas dentro do ambiente organizacional, pois existe a necessidade de a organização voltar-se para seu interior e verificar os meios que Ihe proporcionam vantagens competitivas em relação aos concorrentes, e assim estruturar suas estratégias de acordo com as suas capacidades.

Além disso, um dos principais fatores que mede o nível de competitividade de uma empresa incubada é o capital humano, mais especificamente os níveis de liderança e espírito inovador de empresários e coesão de equipes (Xiao \& Zhao, 2017). Segundo Neneh (2019) personalidade proativa e traço de competitividade de gestores resultam no reconhecimento de oportunidade para a ação empreendedora. Percebe-se então que um melhor entendimento das caraterísticas empreendedoras e suas relações com as tecnologias propostas por novos negócios pode ajudar no incentivo à inovação e no treinamento de empreendedores para aumentar a competitividade de novos empreendimentos (BarNir, 2012).

\subsection{Comportamento Empreendedor}

Empreendedores são como indivíduos inovadores, que visam reformar ou revolucionar o padrão de produção, gerando inovação de processos e de tecnologia por meio da atividade empreendedora. Essa atividade é entendia como a ação empresarial, desenvolvida por indivíduos com características especiais e detentores de habilidades específicas (Schumpeter, 1934). Características empreendedoras são traços de personalidade que distinguem as pessoas detentoras dos mesmos e as tornam mais susceptíveis a adotar um comportamento e atitudes empreendedoras (Schumpeter, 1934; Neneh, 2019).

A análise do comportamento empreendedor demanda a compreensão do contexto no qual ocorre, compreendido como o ambiente econômico, político e cultural, onde são exercidas as atividades empresariais ou de ação empreendedora (Welter, Smallbone, \& Van Gils, 2012). Os autores Hisrich, R. D., Peters, M. P., \& Shepherd (2010) e Neneh (2019) destacam o papel que a intenção ou predisposição desempenha na atividade empreendedora, buscando os fatores motivadores que influenciam no comportamento do indivíduo. Entre os vários fatores, os autores apontam: a educação que se manifesta na capacidade de produzir conhecimento, na habilidade de se comunicar e na aptidão para descobrir as oportunidades e solucionar os problemas; a idade, e sua relação com a carreira de empreendedor, uma vez que a experiência é considerada um dos mais relevantes indicadores para se prever o seu êxito; e o histórico profissional, caracterizado pela experiência profissional prévia do sujeito.

As motivações de uma pessoa a iniciar um negócio tendem a influenciar seu comportamento empreendedor subsequente, ao explorar uma oportunidade de mercado ou uma nova ideia, provavelmente esse indivíduo se engajará em inovações mais diversificadas (Gundolf, Gast, \& Géraudel, 2017). Dessa forma, pressupõe-se que algumas características do processo inovativo devem ser refletidas no desenvolvimento das estruturas organizacionais empreendedoras e ao longo de todas as fases do ciclo organizacional (Asaul \& Faltinskij, 2013). 


\subsection{Ciclo Organizacional do Negócio}

Tanto a competitividade quanto o comportamento empreendedor podem ser analisados em cada fase do ciclo de uma empresa, apresentando-se de forma importante para a evolução nos diferentes estágios. $\mathrm{O}$ ciclo organizacional é similar tanto para indústrias, empresas e produtos, desde sua criação ao término, com estágios intermediários de desenvolvimento, crescimento, maturidade e declínio (Simmonds, 2014). O ciclo de vida dos negócios proposto por Fisk (2008) apresenta sete fases: criar, lançar, estabilizar, expandir, amadurecer, evoluir e sair, conforme Figura 1.

Figura 1

Fases do Negócio de Peter Fisk.

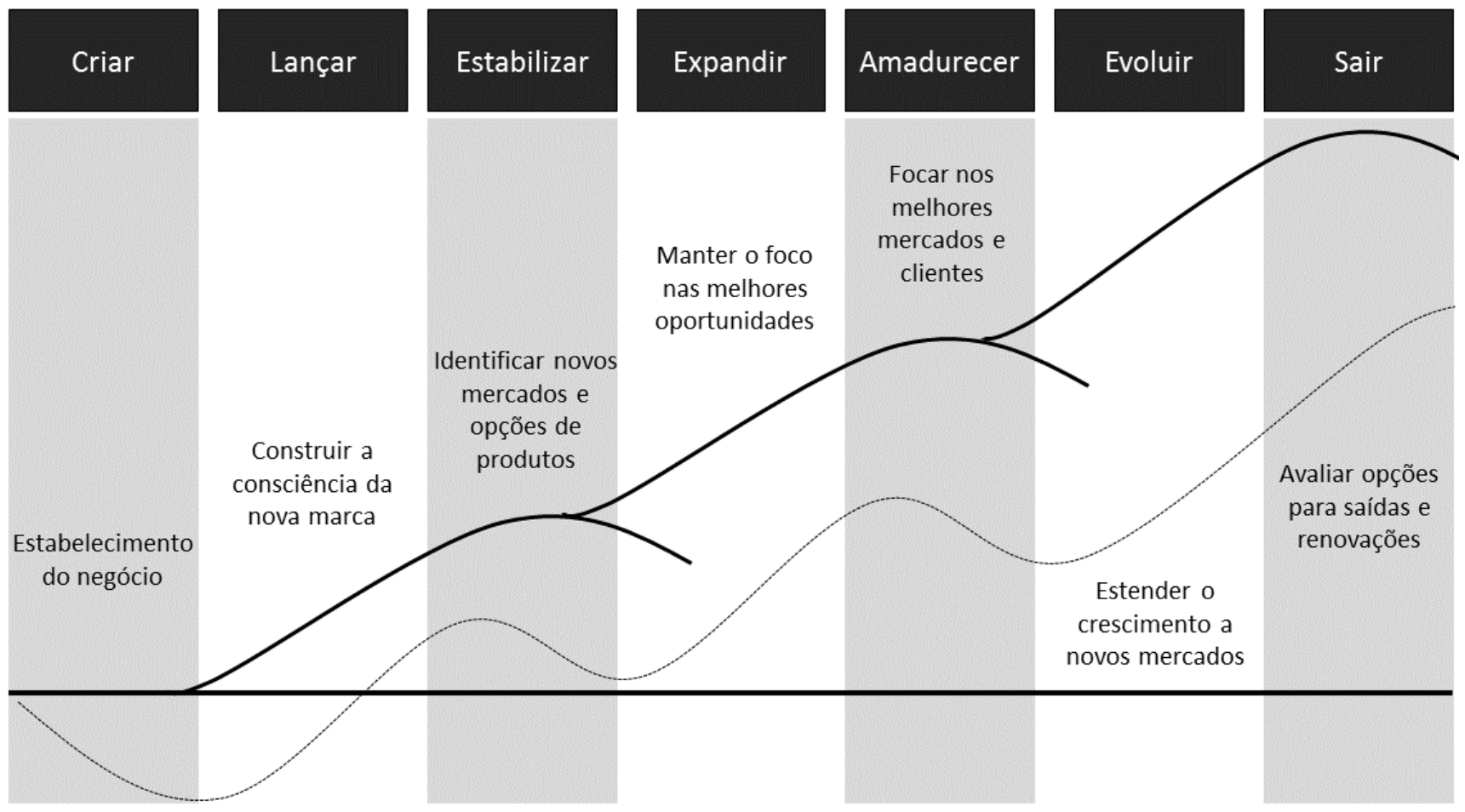

Fonte: Fisk (2008)

O autor explica que as fases se curvam entre momentos de acelerado crescimento, nos quais a inovação e ampliação são importantes, seguidos de períodos, nos quais a empresa precisa reagrupar-se para construir uma nova plataforma para o próximo estágio de crescimento. Dessa forma, compreende-se que o foco e a cultura da organização acabam sendo diferentes conforme a fase organizacional do negócio, e em cada mudança existem novos desafios, inclusive na postura e no comportamento da pessoa que lidera e administra a empresa

$\mathrm{Na}$ abordagem de Fisk (2008) existe a constatação de que a maioria das empresas tem morrido jovem, por tornarem-se vítimas de estratégias fundadas na sua incapacidade de entender o mundo em transformação ou de evoluírem enquanto crescem. Dessa forma, percebe-se que um dos meios para uma empresa conseguir crescer e manter-se competitiva é ter conhecimento em torno dos estágios do seu desenvolvimento para aproveitar as oportunidades e tentar minimizar as dificuldades, esse entendimento na fase inicial fortalece o negócio e ameniza os desafios das próximas etapas (ANPROTEC, 2016).

O processo de incubação também pode alterar as fases do ciclo das empresas significativamente e isso se deve principalmente a quatro fatores: desenvolvimento de credibilidade; encurtamento da curva de aprendizado dos empreendedores; resolução de problemas de forma mais rápida; e acesso a uma rede de relacionamento de empreendedores (ANPROTEC, 2016; Dee, Livesey, Gill, \& Minshall, 2011). 


\subsection{Sistemas de Mensuração de Desempenho}

Os SMDs são instrumentos de suporte que oferecem uma visão ampla dos processos principais e dos níveis de atingimento dos objetivos da organização (Neuenfeldt Júnior, Siluk, Soliman, \& Paris, 2018). Para Elg, Broryd, \& Kollberg (2013), a mensuração de desempenho pode ser identificada em três abordagens: (1) refere-se na perspectiva de contabilidade de gestão que afirma que a medida deve ser integrada no planejamento e orçamento das organizações; (2) aponta a partir de uma perspectiva de operações que identifica a medição de desempenho como conjunto de métricas utilizadas para quantificar a eficiência e a eficácia das ações e; (3) controle estratégico, sendo que a importância é dada a relação entre a gestão superior e as operações, incluindo, a implantação de metas e suas medidas relacionadas a vincular iniciativas estratégicas com o desempenho operacional.

A mensuração de desempenho visa não ser apenas uma ferramenta crucial na elaboração das estratégias empresariais, mas também servir como parâmetro básico da sua correspondência quanto ao contexto holístico externo a fim de tornar elemento importante na administração do negócio (Neuenfeldt, Mairesse Siluk, \& Noal Da Silveira, 2013). Existem vários métodos para mensurar desempenho (Neuenfeldt Júnior, 2014), porém conforme Soliman (2014) eles não devem ser entendidos como modelos isolados, mas sim como opções flexíveis capazes de ajustarem-se da melhor forma possível a realidade que se pretende modelar, cabendo ao usuário da ferramenta a sensibilidade para tanto, uma vez que nem mesmo a literatura cientifica apresenta um consenso de qual o método seja o mais apropriado.

Apesar da mensuração possuir um papel relevante na gestão eficiente e eficaz das organizações, ainda há muito o que se discutir sobre tal temática, que engloba indicadores em todo o processo (Tseng \& Liao, 2015). Nos estudos de Love, Liu, Matthews, Sing, \& Smith (2015) é apontadas quatro funções sobre a construção de um SMD: (1) identificar o sucesso, o nível de satisfação das necessidades dos clientes, onde existem problemas e onde as melhorias são essenciais; (2) entender seus processos e o que eles sabem ou o que eles não sabem; (3) assegurar a adequação de cada decisão tomada; e (4) indicar se os resultados esperados e previstos foram cumpridos.

Sob a luz das características desejáveis para um SMD, destaca-se o método Key Performance Indicators (KPI) visto que sua concepção está alinhada com a proposição desse estudo. Os indicadores KPls são técnicas de gestão empregadas para permitir o monitoramento de negócios eficiente e eficazes, sendo geralmente reconhecidos como um conjunto de medidas críticas para o sucesso atual e futuro de qualquer organização (Graham et al., 2015). Os mesmos autores relatam cinco características que definem um KPI: (1) prestação de contas; (2) facilmente assimilado; (3) temporal; (4) relevantes; e (5) consistentes, atributos essenciais para uma análise. A medição de desempenho pretende ser mais do que uma ferramenta crucial na elaboração de estratégias corporativas, mas também servir como parâmetro básico de sua correspondência no contexto holístico externo para se tornar um elemento importante na gestão empresarial (Neuenfeldt Júnior, Siluk, Soliman, \& Paris, 2018).

Finalizada a segunda parte acerca da fundamentação teórica desse trabalho, o foco recaí para os procedimentos adotados para o alcance do objetivo.

\section{Metodologia}

Essa pesquisa aplicada de natureza teórico-empírica fundamentada no indivíduo, caracteriza-se como exploratória-descritiva, possui uma abordagem quantitativa dos dados e concepção epistemológica positivista. Visando atender ao objetivo da pesquisa, foi desenvolvido um método que compreende diversas etapas, entre elas a definição do cenário da pesquisa com a classificação das startups dentro do ciclo organizacional do negócio e a construção e estruturação do modelo de mensuração. 


\subsection{Definição do cenário da pesquisa}

O universo pesquisado refere-se 31 empresas incubadas que foram selecionadas por meio do método de amostragem não probabilístico por acessibilidade localizadas em incubadoras sediadas em universidades do extremo sul do Rio Grande do Sul, Brasil. A unidade de análise refere-se aos indivíduos ou gestores dessas empresas e os dados primários foram obtidos por meio da aplicação de questionário estruturado, realizado entre os meses de dezembro de 2016 a janeiro de 2018 e pautando-se na abordagem desenvolvida por Fisk (2008).

As empresas pesquisadas pertencem às seguintes incubadoras: Pulsar Incubadora (UFSM) (8), Incubadora Tecnológica da UFSM (6), Incubadora Tecnológica da Universidade de Santa Cruz do Sul (2), Ulbratech da Universidade Luterana do Brasil (2), Incubadora Tecnológica da Universidade Franciscana (9), Incubadora Tecnológica de Caxias do Sul (2), Raiar Pontifícia Universidade Católica do Rio Grande do Sul (2). Pautando-se na amostra total, encontram-se na fase estabilização $29 \%$ (9), fase criação $23 \%$ (8), fase evolução $19 \%$ (5), fase lançamento $16 \%$ (5), fase expansão $10 \%$ (3) e fase amadurecimento $3 \%$. Essa pesquisa objetiva demonstrar os resultados dos gestores da fase criação em relação a mensuração do comportamento empreendedor.

\subsection{A Construção e a Estruturação do Modelo de Mensuração}

O instrumento de coleta propõe afirmações sobre ações do empreendedor respondidas com base na escala adaptada de Likert pautando-se nas seguintes semânticas: 1 (nunca); 2 (raras vezes); 3 (às vezes); 4 (frequentemente); e 5 (sempre). Essa mesma escala foi adaptada com o objetivo de usá-la na metodologia Análise Hierárquica de Processos (AHP), (Saaty, 1991). No instrumento as dez Características do Comportamento Empreendedor (CCEs) foram inseridas em 3 dimensões, denominadas "realização" com 5 características, "planejamento" com 3 características e "poder" com 2 características.

O processo de tabulação e codificação dos dados foram realizados em planilha eletrônica Excel ${ }^{\circledR}$, conferindo possíveis os erros de digitação e ajustando-se a escala invertida do instrumento. Os dados tratados pelo SMD, visaram estimar os KPIs, utilizando os elementos da AHP em uma abordagem multicriterial, onde os critérios foram avaliados de forma pareada.

Na planilha, foram divididas em abas distintas as etapas do modelo de mensuração, desde a inserção dos dados, executando a modelagem até chegar ao resultado final: um painel com filtros, indicando as características empreendedoras de cada gestor por etapa do ciclo organizacional. Por meio da árvore de decisão (Figura 2), o modelo apresenta através do Ponto de Vista Fundamental (PVF), qual é a dimensão mais predominante daquele gestor, bem como através do Fator Critico de Sucesso (FCS), a CCE mais presente baseado no comportamento específico da característica (KPI).

A estruturação da mensuração foi dividida em quatro partes: construção da árvore de decisão; construção dos indicadores de desempenho e escalas de avaliação; validação dos indicadores e escalas de avaliação; construção dos instrumentos de avaliação, etapas essas sugeridas pela AHP. A estrutura denominada árvore de decisão foi elaborada a partir do instrumento compreendido por 55 assertivas de comportamento empreendedor distribuídas em 3 dimensões e 10 características. Destaca-se que do total das assertivas, 5 delas correspondem ao fator de correção e não foram usadas nos cálculos. Dentro do modelo cada assertiva corresponde a um KPI, cada KPI tem um peso correspondente ao FCS e cada FCS faz correspondência ao PVF. A Figura 2 representa a estrutura hierárquica para avaliação da competitividade das startups à luz do comportamento empreendedor do gestor. 
Figura 2

Árvore de Decisão com a Estrutura Hierárquica

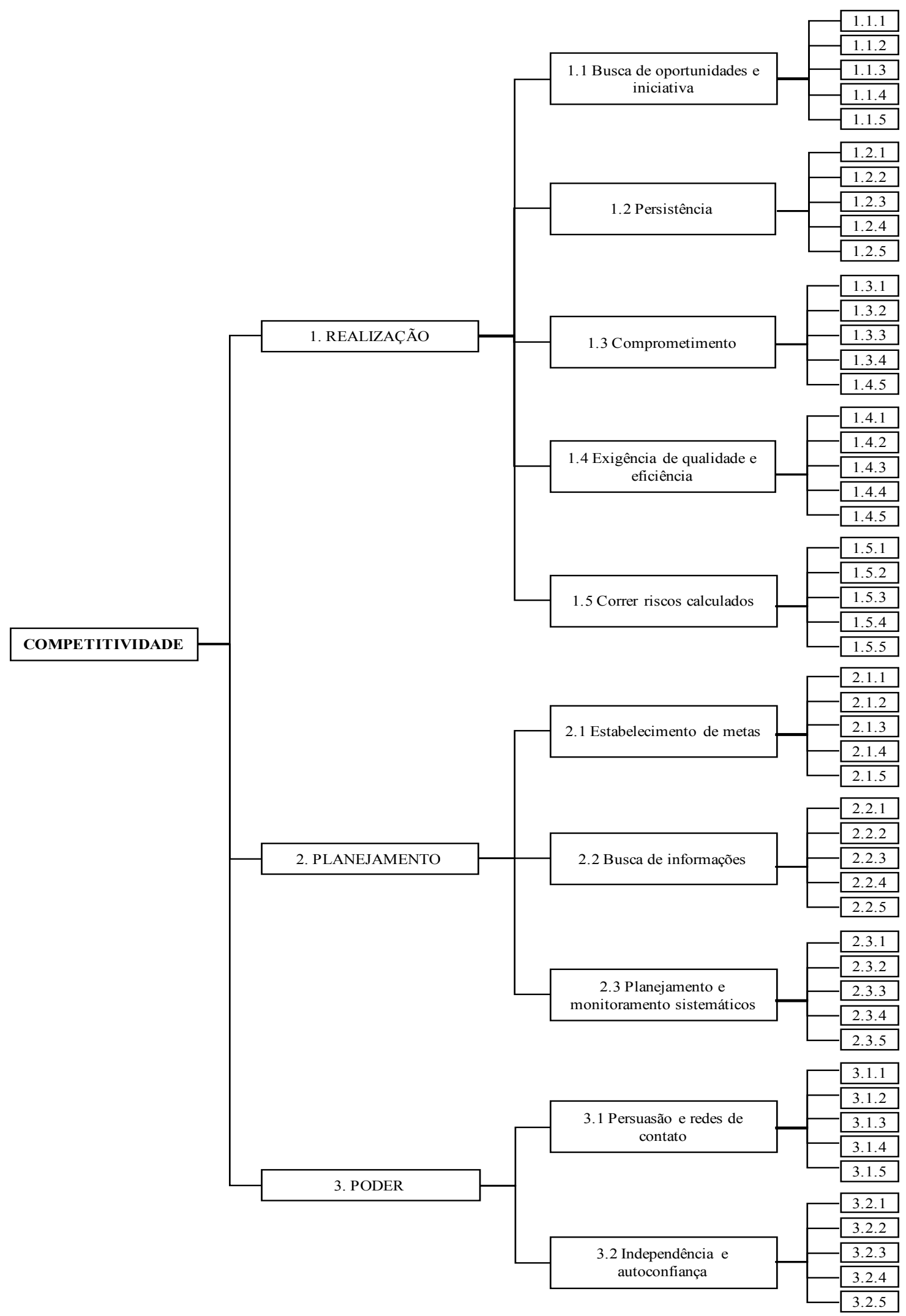

Fonte: Adaptação de Saaty (1991); com base em McClelland (1987);

United Nations Conference on Trade and Development [UNCTAD], (n.d.); Vilas Boas (2015) 
O painel inicial do modelo de mensuração, apresentado na Figura 3, permitiu ao usuário acessar o dashboard e a compilação dos resultados das empresas pesquisadas.

Figura 3

Painel de Controle do Modelo de Mensuração Desenvolvido

\section{\& NIC Ferramenta para Avaliação de Competitividade na perspectiva do Comportamento Empreendedor}

Selecione a opção desejada:

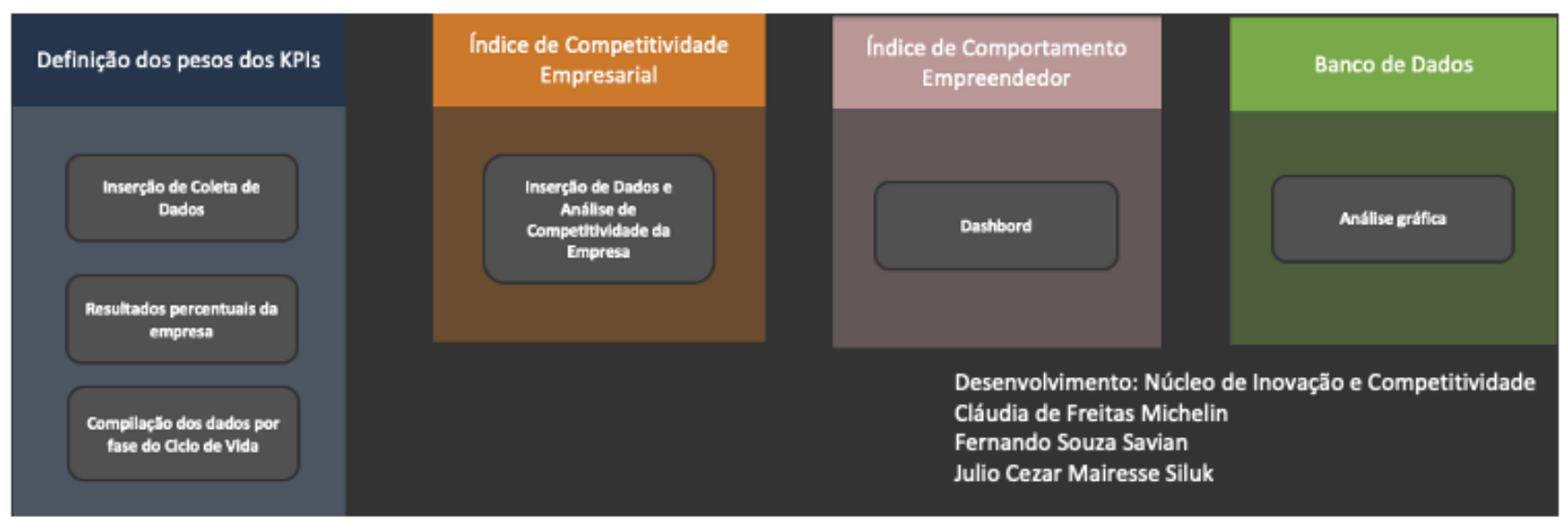

Fonte: Michelin (2018)

No primeiro quadro do dashboard são inseridos os dados de todas as coletas e o modelo faz a compilação das empresas em cada etapa do ciclo organizacional do negócio. Nesta fase são gerados dados individuais por empresa, gerando um peso relativo a cada KPI e esse peso é expandido na estrutura hierárquica. Para cada etapa do ciclo organizacional foi gerada uma estrutura hierárquica. O modelo converteu a escala likert de 5 pontos do instrumento para a escala da AHP. O Painel traz ainda informações sobre o índice de competitividade empresarial, que é relativo ao índice de comportamento empreendedor do gestor investigado e um banco de dados com análises gráficas.

Após inserir os dados das respostas dos gestores e respectivos pesos da AHP, a ferramenta computacional desenvolvida fez a compilação dos dados por empresa dentro da respectiva fase do ciclo organizacional em que ela se encontra. Na aba de compilação dos dados aparecem todas as empresas de cada uma das fases. As etapas iniciais de inserção de dados de cada respondente foram necessárias para calibrar os dados e para gerar os cálculos. A partir de então, fez-se novamente a inserção dos dados por empresa para que fosse gerado um banco de dados com o índice de comportamento empreendedor de cada gestor. Salienta-se que quando é gerado o banco de dados, também são gerados gráficos demonstrativos daquela informação. Para essa pesquisa adotouse um escore para apoiar as análises no sentido de demonstrar a intensidade das CCEs nos gestores conforme a Tabela 1.

Tabela 1

Intensidade dos Níveis das Características do Comportamento Empreendedor

\begin{tabular}{c|c|c}
\hline Níveis & Descrição & Intensidade da característica CCE \\
\hline N1 & $0 \%$ a $50 \%$ & Inexistente \\
\hline N2 & $50,01 \%$ a $62,50 \%$ & Baixa \\
\hline N3 & $62,51 \%$ a $75,00 \%$ & Moderada \\
\hline N4 & $75,01 \%$ a $87,50 \%$ & Alta \\
\hline N5 & $87,51 \%$ a $100 \%$ & Plena \\
\hline
\end{tabular}

Fonte: Autores 
A partir da definição dos fatores críticos de sucesso levantados conforme árvore hierárquica, foi necessária a construção das escalas de avaliação para ser possível mensurar o comportamento e isso significar competitividade. O primeiro passo nessa construção foi o entendimento de cada FCS para evitar possíveis interpretações dúbias por parte dos respondentes. O segundo passo foi desdobrar cada FCS em cinco possíveis níveis de resposta (N1, N2, N3, N4 e N5), onde em cada caso o nível "N1" corresponde à pior situação possível para a competitividade, enquanto o nível "N5" é considerado como a mais favorável possível. O ponto médio "N3", por sua vez, diz respeito ao desempenho médio geral.

Para afirmar que os índices de comportamento empreendedor correspondem a competitividade das startups, o estudo comparou os índices de outras pesquisas desenvolvidas em conjunto com o mesmo grupo de empresas e a mesma temática, competitividade a partir de outras perspectivas. A avaliação dessa correlação, conforme a Tabela 2, evidencia as faixas de avaliação da competitividade desenvolvidas pelo NIC (Núcleo de Inovação e Competitividade, 2015).

Tabela 2

Faixas de Avaliação da Competitividade

\begin{tabular}{ccc}
\hline Nível & Descrição dos níveis de avaliação & Descrição da competitividade \\
\hline N1 & Entre $0-24,9 \%$ & Não competitiva \\
\hline N2 & Entre $25 \%-49,9 \%$ & Pouco competitiva \\
\hline N3 & Entre $50 \%-74,90 \%$ & Potencialmente competitiva \\
\hline N4 & Entre $75 \%-100 \%$ & Plenamente competitiva \\
\hline & Fonte: Núcleo de Inovação e Competitividade (2015)
\end{tabular}

O estudo utilizou a escala de avaliação de competitividade definidos na tabela, observando o enquadramento das empresas pesquisadas em relação as faixas de avaliação da competitividade e o nível de intensidade das CCEs.

\section{Resultados e Discussões}

Os dados gerados pelo modelo de mensuração iniciou pela análise geral de comportamento empreendedor presente nas diferentes fases do ciclo organizacional do negócio. Os resultados aqui descritos são referentes exclusivamente as empresas que foram classificadas na fase inicial do negócio, denominada fase de criação.

Foram verificados por meio dos índices das estruturas hierárquicas os KPIs mais preponderantes em cada FCS, de modo que foi possível identificar as características do comportamento empreendedor com maior índice de comportamento e no mesmo sentido identificar qual foi o PVF mais presente. A identificação dessas características permitiu avaliar, dentro da fase de criação, os comportamentos e características mais preponderantes dentre as dimensões realização, planejamento e poder, ressaltando que segundo Xiao \& Zhao (2017) o capital humano é um dos fatores capazes de medir o nível de competitividade de uma empresa incubada. A Tabela 3 apresenta resultados da etapa de criação para a dimensão realização, composta de cinco FCS.

A primeira coluna da Tabela 3 diz respeito ao número da assertiva de acordo com o instrumento aplicado. A segunda coluna descreve o comportamento empreendedor em ordem decrescente dos resultados do KPI e a terceira demonstra o percentual do FCS, que quando somado aos demais comportamentos chega-se ao percentual total da CCE. A quarta coluna demonstra o resultado relativo do FCS, representado por um índice formado pelas respostas dos gestores da fase criação, uma vez que é sabido que o total máximo desse índice é cinco e que ele foi estimado de acordo com o peso da característica em relação a soma total da estrutura hierárquica desenvolvida para o modelo. A próxima etapa do cálculo é a proporcionalidade desse índice em relação ao PVF, chegando-se ao resultado final, que é o percentual do comportamento empreendedor. 
Tabela 3

FCS 1.1 Busca de Oportunidade e Iniciativa - Fase Criação

\begin{tabular}{|c|c|c|c|c|}
\hline Cód. & KPI (assertivas) & $\begin{array}{l}\text { Resultado } \\
\text { KPI }\end{array}$ & $\begin{array}{l}\text { Resultado } \\
\text { FCS }\end{array}$ & Peso \\
\hline 1 & 1.1.1 Esforço-me para realizar as coisas que devem ser feitas. & $30,00 \%$ & \multirow{5}{*}{4,648477} & \multirow{5}{*}{$20 \%$} \\
\hline 23 & 1.1.3 Gosto de desafios e novas oportunidades. & $30,00 \%$ & & \\
\hline 12 & $\begin{array}{l}\text { 1.1.2 Faço as coisas que devem ser feitas sem que os outros } \\
\text { tenham que me pedir. }\end{array}$ & $18,58 \%$ & & \\
\hline 45 & $\begin{array}{l}\text { 1.1.5 Aventuro-me a fazer coisas novas e diferentes das que fiz } \\
\text { no passado. }\end{array}$ & $12,71 \%$ & & \\
\hline 34 & $\begin{array}{l}\text { 1.1.4 Prefiro executar tarefas que domino perfeitamente e em } \\
\text { que me sinto seguro. }\end{array}$ & $1,68 \%$ & & \\
\hline Total & - & $92,97 \%$ & - & - \\
\hline
\end{tabular}

Fonte: Autores

Nesse mesmo formato foram realizadas tabelas para todos os dez FCSs das dimensões realização, planejamento e poder e um gráfico geral para cada uma delas. O Gráfico 1 apresenta a compilação dos resultados da fase criação para os 5 FCS apresentados da dimensão realização.

Gráfico 1

Resultados dos FCSs da Dimensão Realização - Fase Criação

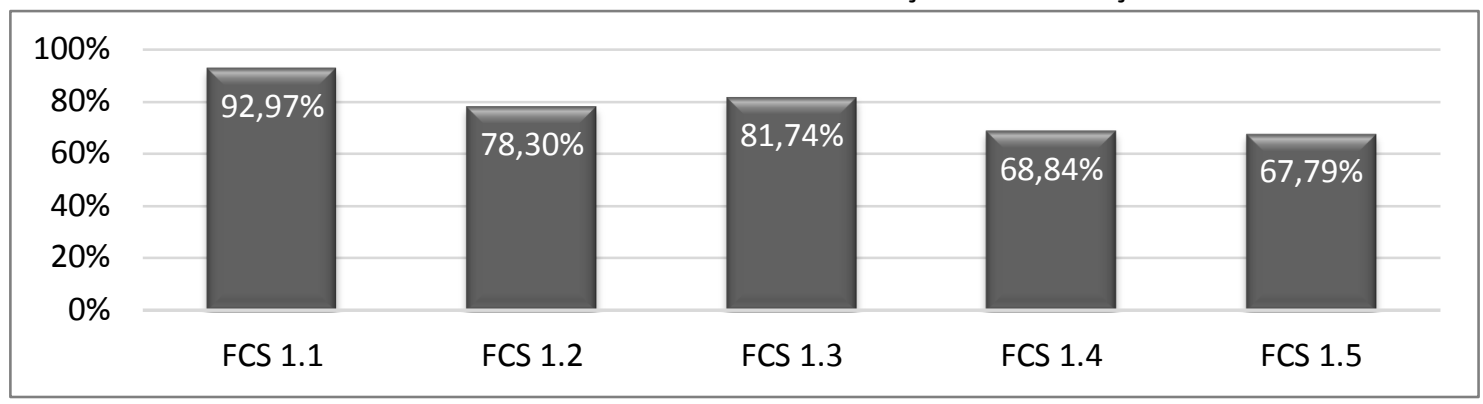

Fonte: Autores

É possível perceber no gráfico que dois FCS foram considerados elevados em relação a intensidade do comportamento empreendedor, FCS 1.2: Persistência e FCS 1.3: Comprometimento. A característica comprometimento apresentou um índice igual a $81,74 \%$, demonstrando nos resultados que os gestores nessa fase são fiéis as promessas que fazem e frequentemente terminam suas atividades a tempo. Esse último indicador vai ao encontro do que diz a UNCTAD (n.d.) de que o indivíduo empreendedor deve desenvolver procedimentos para assegurar que o trabalho atenda aos padrões de qualidade previamente combinados e que seja finalizado a tempo. Já o FCS 1.1: Busca de Oportunidade e Iniciativa foi considerado intensidade plena, uma vez que os gestores pesquisados nesta fase encontram-se no momento de criação, momento esse de se ter iniciativa, e nesse sentido a predisposição para iniciar algo é fundamental (Neneh, 2019). O Gráfico 2 apresenta a compilação dos resultados da fase criação para os 3 FCS apresentados da dimensão planejamento.

Gráfico 2

Resultados dos FCSs da Dimensão Planejamento - Fase Criação

\begin{tabular}{|c|c|c|c|}
\hline \\
\hline $85 \%$ & & & $88,16 \%$ \\
\hline \multirow{2}{*}{$\begin{array}{l}80 \% \\
75 \%\end{array}$} & $82,49 \%$ & $85,45 \%$ & \\
\hline & FCS 2.1 & FCS 2.2 & FCS 2.3 \\
\hline
\end{tabular}

Fonte: Autores 
Todas as três características da dimensão planejamento apresentaram índices acima de quatro e percentuais altos de comportamento empreendedor. Para Fisk (2008) e Tripathi, Oivo, Liukkunen, \& Markkula, (2019) a criação de um novo negócio é impulsionado pela ambição empreendedora, o que possibilita aos gestores colocarem em prática as suas ideias. Para Frota, Brasil, \& Fontenele (2014) empreendedores são impulsionados pela necessidade de sobrevivência, de concretização de uma ideia ou simplesmente pelo desejo de se tornarem donos do próprio negócio. Os gestores investigados possuem essa ambição e procuram condições favoráveis para a concretização de sua ideia, o simples fato de participar de um edital de processo seletivo para inserção em uma incubadora já demonstra essa vontade de empreender e se destacar no mercado. O Gráfico 3 apresenta a compilação dos resultados da fase criação para os 2 FCS apresentados da dimensão poder.

Gráfico 3

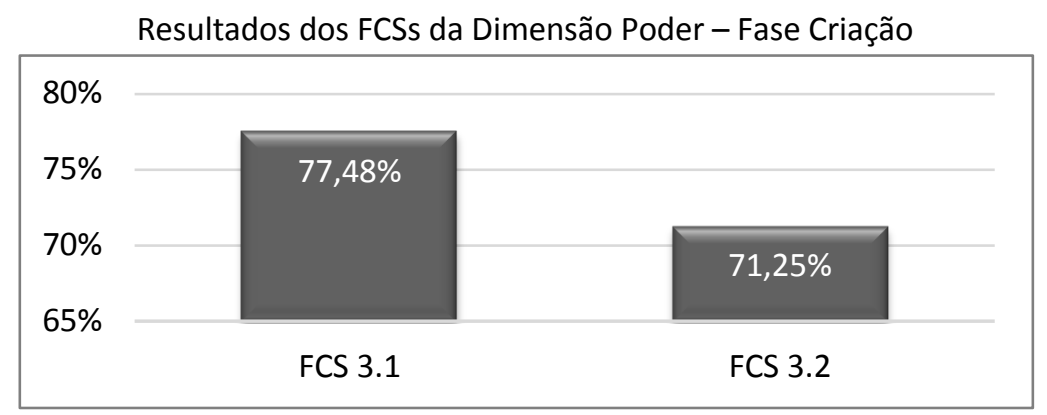

Fonte: Autores

O último bloco das características do comportamento empreendedor refere-se ao PVF 3 dimensão poder dividida no FCS 3.1 Persuasão e rede de contato e FCS 3.2 Independência e autoconfiança. Características de poder estão associadas a necessidade de poder, conforme McClelland (1987), uma vez que pessoas empreendedoras encontram meios de influenciar outras pessoas (Coan, 2011). Relacionado ao comando, ao desejo de ser forte, o último autor enfatiza que o poder leva a situações de competições, disputas e status, portanto essa necessidade leva as pessoas a correrem riscos consideráveis, uma vez que as mesmas são mais preocupadas com prestígio e influência do que com o desempenho eficaz.

Em relação a dimensão planejamento, a categoria poder manteve-se abaixo, o FCS 3.2 Independência e Autoconfiança atingiu $72,25 \%$, intensidade moderada de CCE, mas na correlação com a competitividade significa a empresa potencialmente competitiva (NIC, 2015). Analisando todos os 10 FCS da fase criação, 50\% deles obtiveram índices acima de 4 e os outros 50\% acima de 3, significando que embora três características apresentem intensidade moderada, todas as demais, representando a maioria, apresentaram-se com intensidade alta e uma com intensidade plena, entendendo que muitos comportamentos são presentes nos gestores dessa fase de criação dos negócios. Os PVFs 1 e 2, representando as dimensões realização e planejamento, respectivamente, obtiveram índices mais altos. Esse resultado reflete os comportamentos que mais correspondem a essa primeira fase do ciclo organizacional de criar um negócio, nas quais a necessidade de realização se faz muito presente bem como a vontade de planejamento e atingimento de metas, que segundo Neneh (2019) são características indispensáveis do empreendedor.

Importante resgatar nas análises a importância de desenvolver tais características empreendedoras. Para os autores Engelman \& Fracasso (2013) e Raupp \& Beuren (2011), o conjunto dessas características permite instigar o esforço do indivíduo, denominado empreendedor no desenvolvimento de alguma ideia, que consequentemente poderá culminar na criação de um negócio. Os autores destacam ainda que nem todas as pessoas possuem habilidades que permitem empreender, mas que algumas são inerentes ao indivíduo e outras possam ser aprendidas. 
Salienta-se ao final da análise de dados quantitativos que todos os KPIs interferiram na competitividade das empresas na perspectiva do comportamento empreendedor do gestor. Conforme Michelin (2018), alguns predominaram mais no resultado, outros menos, alguns comportamentos foram muito evidentes na fase de criação, o que pode ter relação com o momento daquele gestor em relação ao seu negócio, pois evidenciou-se que o fator comportamento influencia na fase e isso também pode ser definido como um direcionador de competitividade, o que corrobora com o estudo de Neneh (2019). A seguir as conclusões do estudo.

\section{Conclusões}

Essa pesquisa teve como objetivo mensurar o comportamento empreendedor de gestores na fase inicial do ciclo organizacional em startups incubadas, enfatizando que o resultado da mensuração pode ser norteador de competitividade. Os resultados fizeram referencia as empresas que se classificaram na fase de criação do negócio, por entender ser essa uma etapa crucial e decisiva na geração de uma nova ideia e na motivação de colocá-la em prática.

O modelo de mensuração desenvolvido e apresentado neste estudo foi testado satisfatoriamente nas empresas, uma vez que os dados inseridos em uma árvore hierárquica de apoio a decisão indicaram que a maioria das startups se enquadra em índices potencialmente e plenamente competitivos correlacionando com os níveis de intensidade de comportamento empreendedor. Dessa forma, apesar de estarem em fases relativamente iniciais em seus negócios, os gestores investigados já se inserem de forma competitiva nos mercados que se propõem a atuar.

Analisando a relação entre os níveis das faixas de intensidade de CCE com as faixas de avaliação da competitividade, pode-se afirmar que as startups em seu estágio inicial foram consideradas competitivas porque seus gestores apresentam intensidade relevante de CCEs. Nesse sentido a competitividade na perspectiva comportamental é um norteador de competividade, constatação que vai ao encontro do posicionamento de vários autores que estudam essas temáticas.

A mensuração desenvolvida além de permitir aos pesquisadores identificar o nível de competitividade da empresa a partir do índice de comportamento empreendedor do gestor, possibilitou identificar quais indicadores apresentam o maior potencial de melhoria, além de auxiliar no processo de tomada de decisão ampliando o diagnóstico estratégico organizacional, fator que contribui para a competitividade. Dessa forma, os gestores das startups incubadas passam a adquirir maior conhecimento de como suas atitudes e seus comportamentos afetam o posicionamento competitivo da empresa sob seu comando.

Destaca-se também a relevância de desenvolver sistemas de mensuração de desempenho, uma vez que esse método proporciona uma visão sistêmica dos critérios de avaliação retornando as empresas uma resposta mais rápida de alteração e ou melhorias do que está sendo investigado, além também do dinamismo e flexibilidade que oferece.

Os resultados são promissores no âmbito de empresas de tecnologia incubadas, podendo ser testado em outras empresas para fins de pesquisas futuras. Sugere-se estudos de comparação entre todas as fases do ciclo organizacional, no sentido de verificar quais são as CCEs mais presentes nas fases iniciais, de meio e finais de um ciclo, bem como identificar as ausentes ou em menor intensidade. Essas informações são relevantes para o autoconhecimento dos gestores além também de valorar pesquisas sobre mensuração de aspectos intangíveis como contribuição para o ambiente corporativo e para novos achados e estudos. 


\section{Referências bibliográficas}

ANPROTEC, A. N. de E. P. de E. I. (2016). Estudo de impacto econômico: segmento de incubadoras de empresas do Brasil. Retrieved from www.anprotec.org.br

Antonenko, P. D., Lee, B. R., \& Kleinheksel, A. J. (2014). Trends in the crowdfunding of educational technology startups. TechTrends, 58(6), 36-41. https://doi.org/10.1007/s11528-014-0801-2

Asaul, V., \& Faltinskij, R. (2013). Improvement of entrepreneur entities' competitiveness through innovative behaviour. World Applied Sciences Journal, 23(13), 60-64. https://doi.org/10.5829/idosi.wasj.2013.23.pac.90013

BarNir, A. (2012). Starting technologically innovative ventures: Reasons, human capital, and gender. Management Decision, 50(3), 399-419. https://doi.org/10.1108/00251741211216205

Bartz, T., Siluk, J. C. M., \& Bartz, A. P. B. (2014). Improvement of industrial performance with TPM implementation. Journal of Quality in Maintenance Engineering, 20(1), 2-19. https://doi.org/10.1108/JQME-07-2012-0025

Bird, B., Schjoedt, L., \& Baum, J. R. (2012). Editor's Introduction. Entrepreneurs' Behavior: Elucidation and Measurement. Entrepreneurship Theory and Practice, 36(5), 889-913. https://doi.org/10.1111/j.15406520.2012.00535.x

Caseiro, N., \& Coelho, A. (2018). Business intelligence and competitiveness: the mediating role of entrepreneurial orientation. Competitiveness Review, 28(2), 213-226. https://doi.org/10.1108/CR-092016-0054

Coan, M. (2011). Educação para o Empreendedorismo: implicações epistemológicas, políticas e práticas (Universidade Federal de Santa Catarina). https://doi.org/10.16194/j.cnki.31-1059/g4.2011.07.016

Colombo, M. G., \& Grilli, L. (2005). Founders' human capital and the growth of new technology-based firms: A competence-based view. Research Policy, 34(6), 795-816. https://doi.org/10.1016/j.respol.2005.03.010

Colombo, M. G., \& Piva, E. (2012). Firms' genetic characteristics and competence-enlarging strategies: A comparison between academic and non-academic high-tech start-ups. Research Policy, 41(1), 79-92. https://doi.org/10.1016/j.respol.2011.08.010

Costa, R., Siluk, J., Neuenfeldt Júnior, A., Soliman, M., \& Nara, E. (2015). The management of industrial competitiveness through the application of methods up and multi-criteria in a bovine slaughterhouse. Ingeniare, 23(3), 383-394. https://doi.org/10.4067/S0718-33052015000300007

Dee, N. J., Livesey, F., Gill, D., \& Minshall, T. (2011). Incubation for Growth A review of the impact of business incubation on new ventures with high growth potential. Retrieved from www.nesta.org.uk

Díaz-Chao, Á., Sainz-González, J., \& Torrent-Sellens, J. (2016). The competitiveness of small network-firm: A practical tool. Journal of Business Research, 69(5), 1769-1774.

https://doi.org/10.1016/j.jbusres.2015.10.053

Dutta, S., \& Folta, T. B. (2016). A comparison of the effect of angels and venture capitalists on innovation and value creation. Journal of Business Venturing, 31(1), 39-54.

https://doi.org/10.1016/j.jbusvent.2015.08.003 
Elg, M., Broryd, K. P., \& Kollberg, B. (2013). Performance measurement to drive improvements in healthcare practice. International Journal of Operations and Production Management, 33(11), 1623-1651. https://doi.org/10.1108/IJOPM-07-2010-0208

Engelman, R., \& Fracasso, E. M. (2013). Contribuição das incubadoras tecnológicas na internacionalização das empresas incubadas. Revista de Administração, 48(1), 165-178. https://doi.org/10.5700/rausp1080

Figueiredo, E., \& Paiva, T. (2019). Senior entrepreneurship and qualified senior unemployment: The case of the Portuguese Northern region. Journal of Small Business and Enterprise Development, 26(3), 342-362. https://doi.org/10.1108/JSBED-01-2018-0006

Fisk, P. (2008). Business Genius : a More Inspired Approach to Business Growth. Retrieved from https://www.wiley.com/en-br/Business+Genius:+A+More+Inspired+Approach+to+Business+Growth-p9781841127903

Frota, G. B. da, Brasil, M. V. D. O., \& Fontenele, R. E. S. (2014). Influência das Características Socioeconômicas, Capacidade de Gestão e Comportamento Empreendedor no Sucesso dos Empreendedores Participantes do Programa de Microcrédito do Banco Palmas. REGEPE - Revista de Empreendedorismo e Gestão de Pequenas Empresas, 3(2), 42-69. https://doi.org/10.14211/regepe.v3i2.140

GEM: Global Entrepreneurship Monitor. (n.d.). https://doi.org/10.5860/choice.51-4537

Graham, I., Goodall, P., Peng, Y., Palmer, C., West, A., Conway, P., ... Dettmer, F. U. (2015). Performance measurement and KPIs for remanufacturing. Journal of Remanufacturing, 5(1), 1-17. https://doi.org/10.1186/s13243-015-0019-2

Gruber, M., \& MacMillan, I. C. (2017). Entrepreneurial Behavior: A Reconceptualization and Extension Based on Identity Theory. Strategic Entrepreneurship Journal, 11(3), 271-286. https://doi.org/10.1002/sej.1262

Gundolf, K., Gast, J., \& Géraudel, M. (2017). Startups' Innovation behaviour: an investigation into the Role of entrepreneurial motivations. International Journal of Innovation Management, 21(7). https://doi.org/10.1142/S1363919617500542

Hahn, G. J. (2020). Industry 4.0: a supply chain innovation perspective. International Journal of Production Research, 58(5), 1425-1441. https://doi.org/10.1080/00207543.2019.1641642

Hisrich, R. D., Peters, M. P., \& Shepherd, D. A. (2010). Entrepreneurship. Retrieved from https://repository.iimb.ac.in/handle/2074/12345

Kosuri, P. (2017, December). Democratizing Entrepreneurship: Online Documents, Tools, and Startup KnowHow.

Love, P. E. D., Liu, J., Matthews, J., Sing, C. P., \& Smith, J. (2015). Future proofing PPPs: Life-cycle performance measurement and Building Information Modelling. Automation in Construction, 56, 26-35. https://doi.org/10.1016/j.autcon.2015.04.008

Marinho, S. V., \& Cagnin, C. (2014). The roles of FTA in improving performance measurement systems to enable alignment between business strategy and operations: Insights from three practical cases. Futures, 59, 5061. https://doi.org/10.1016/j.futures.2014.01.015

Maune, A. (2014). Competitive intelligence and firm competitiveness: An overview. Corporate Ownership and Control, 12(1CONT6), 533-542. https://doi.org/10.22495/cocv12i1c6p1 
McClelland, D. C. (1987). Characteristics of Successful Entrepreneurs. The Journal of Creative Behavior, 21(3), 219-233. https://doi.org/10.1002/j.2162-6057.1987.tb00479.x

Michelin, C. de F. (2018). Modelo de mensuração da competitividade em empresas de base tecnológica na perspectiva do comportamento empreendedor do gestor diante do ciclo organizacional do negócio (Universidade Federal de Santa Maria). Retrieved from https://repositorio.ufsm.br/bitstream/handle/1/18789/TES_PPGADMINISTRACAO_2018_MICHELIN_CLAU DIA.pdf?sequence=1\&isAllowed=y

Minello, I. F. (2010). Resiliência e insucesso empresarial: Um estudo exploratório sobre o comportamento resiliente e os estilos de enfrentamento do empreendedor em situações de insucesso empresarial, especificamente em casos de descontinuidade do negócio (Universidade Federal de São Paulo (USP)). https://doi.org/10.11606/T.12.2010.tde-24062010-114250

Mitsakis, F. V. (2017). Employees' perspectives on strategic human resource development before and after the global financial crisis: evidence from the Greek banking sector. International Journal of Training and Development, 21(4), 285-303. https://doi.org/10.1111/ijtd.12112

Nascimento, F. M. do; Neuenfeldt Júnior, A.; De Paris, S.; Stieler, G.; De Moreira, L. A. (2019) The organizational performance measurement environmental for opening a beverages distributor. Espacios 40(35),6. https://www.revistaespacios.com/a19v40n35/a19v40n35p06.pdf

Neamţu, D. M., Bejinaru, R., \& Hapenciuc, C. V. (2020). Challenges and opportunities for creative-innovative clusters partnerships. Proceedings of the International Conference on Business Excellence, 14(1), 10571070. https://doi.org/10.2478/picbe-2020-0100

Neneh, B. N. (2019). From entrepreneurial alertness to entrepreneurial behavior: The role of trait competitiveness and proactive personality. Personality and Individual Differences, 138, 273-279. https://doi.org/10.1016/j.paid.2018.10.020

Neuenfeldt, A. L., Mairesse Siluk, J. C., \& Noal Da Silveira, M. (2013). Modelo de avaliação de desempenho para o sistema de franquias do Brasil. Cuadernos de Administracion, 26(47), 285-307. Retrieved from http://www.scielo.org.co/pdf/cadm/v26n47/v26n47a12.pdf

Neuenfeldt Júnior, A. L. (2014). Modelagem Para a Mensuração De Desempenho Dos Sistemas Bus Rapid Transit No Brasil. In Dissertação de mestrado (p. 91). https://doi.org/10.13140/RG.2.2.24141.33765

Neuenfeldt Júnior, A. L., Siluk, J. C. M., Soliman, M., \& Paris, S. De. (2018). Modelling for performance measurement of bus rapid transit systems in Brazil. International Journal of Logistics Systems and Management, 30(3), 283. https://doi.org/10.1504/ijlsm.2018.092611

Nora, L. D. D., Siluk, J. C. M., Júnior, A. L. N., Soliman, M., Nara, E. O. B., \& Furtado, J. C. (2016). The performance measurement of innovation and competitiveness in the telecommunications services sector. International Journal of Business Excellence, 9(2), 210-224. https://doi.org/10.1504/IJBEX.2016.074844

Núcleo de Inovação e Competitividade. (2015). Projeto de mensuração de ativos intangíveis em empresas de base tecnológica. Santa Maria - RS.

Peña, I. (2002). Intellectual capital and business start-up success. Journal of Intellectual Capital, 3(2), 180-198. https://doi.org/10.1108/14691930210424761 
Porter, M. E., \& Kramer, M. R. (2011). Creating shared value. Harvard Business Review, 89(62-77). https://doi.org/10.32591/coas.ojss.0201.04037b

Poveda-Bautista, R., García-Melón, M., \& Baptista, D. C. (2013). Competitiveness measurement system in the advertising sector. SpringerPlus, 28(2), 213-226. https://doi.org/10.1186/2193-1801-2-438

Raupp, F. M., \& Beuren, I. M. (2011). Perfil do suporte oferecido pelas incubadoras brasileiras às empresas incubadas. REAd. Revista Eletrônica de Administração (Porto Alegre), 17(2), 330-359. https://doi.org/10.1590/s1413-23112011000200002

Saaty, T. L. (1991). Método de análise hierárquica. Método de Análise Hierárquica, 111. Retrieved from https://sebodomessias.com.br/livro/administracao/metodo-de-analise-hierarquica.aspx

Scarano, T. F., Siluk, J. C. M., Nara, E. O. B., Neuenfeldt Júnior, A. L., \& Da Fontoura, F. B. B. (2014). Diagnóstico do desempenho organizacional em empresas do setor metal mecânico. Espacios, 35(3), 18. https://www.revistaespacios.com/a14v35n03/14350319.html

Schumpeter, J. A. (1934). The Theory of Economic Development. In The Theory of Economic Development. https://doi.org/10.4324/9781315135564

Siluk, J. C. M. (2007). Modelo de Gestão Organizacional com Base em um Sistema de Avaliação de Desempenho (Universidade Federal de Santa Catarina). Retrieved from https://repositorio.ufsc.br/xmlui/bitstream/handle/123456789/90343/247968.pdf?sequence=1\&isAllowe $d=y$

Siluk, J. C. M., Garlet, T. B., Marcuzzo, R., Michelin, C. de F., \& Minello, I. F. (2018). Empreendedorismo de base tecnológica no sul do Brasil. Rev. Administração ReA UFSM, 11, 471-488. https://doi.org/10.5902/19834659

Silveira, O. F. da, Michelin, C. D. F., \& Siluk, J. C. M. (2017). Aplicação de uma proposta para a medição de desempenho de um sistema de gestão da qualidade. Revista de Administração Da UFSM, 10(10), 92. https://doi.org/10.5902/1983465924828

Simmonds, P. (2014). Organizational Life Cycle. In Wiley Encyclopedia of Management. https://doi.org/10.1002/9781118785317.weom120149

Soliman, M. (2014). Avaliação da competitividade em indústrias de transformação de plástico. Universidade Federal de Santa Maria, Santa Maria - RS.

Tripathi, N., Oivo, M., Liukkunen, K., \& Markkula, J. (2019). Startup ecosystem effect on minimum viable product development in software startups. Information and Software Technology, 114, 77-91. https://doi.org/10.1016/j.infsof.2019.06.008

Tseng, P. H., \& Liao, C. H. (2015). Supply chain integration, information technology, market orientation and firm performance in container shipping firms. International Journal of Logistics Management, 26(1), 82-106. https://doi.org/10.1108/IJLM-09-2012-0088

United Nations Conference on Trade and Development, U. (n.d.). Empretec Programme The Entrepreneur's Guide Contents. Retrieved from https://empretec.unctad.org/wpcontent/uploads/2015/09/EG_eng.compressed.pdf 
Vilas Boas, E. P. (2015). O comportamento do empreendedor e suas influências no processo de criação e no desempenho da empresa (Biblioteca Digital de Teses e Dissertações da Universidade de São Paulo). https://doi.org/10.11606/T.12.2015.tde-23062015-081259

Welter, F., Smallbone, D., \& Van Gils, A. (2012). Entrepreneurial processes in a changing economy: Frontiers in European entrepreneurship research. In Entrepreneurial Processes in a Changing Economy: Frontiers in European Entrepreneurship Research. https://doi.org/10.4337/9781781004739

Xiao, J., \& Zhao, Y. (2017). Key Intellectual Capital Factors of Competitiveness for Startups: Evidence from China. Proceedings of the 14th international conference on intellectual capital, knowledge management \& organisational learning (ICICKM 2017), 301-309. Retrieved from http://www.saic.gov.cn/zwgk/tjzl/zhtj/xxzx/201701/t20170119_174517.html

Esta obra está bajo una Licencia Creative Commons Attribución-NoCommercial 4.0 International

\section{(cc) EY-NC}

